\title{
On Solving Boundary Value Problems Associated with Generalised LQ Control of 2-D Systems
}

\author{
Lorenzo Ntogramatzidis Michael Cantoni
}

\begin{abstract}
This paper considers the bounded-frame linear quadratic (LQ) optimal control problem for Roesser models with double-ended constraints on the horizontal and vertical semistates. Necessary and sufficient conditions for optimality are presented for this problem. We also identify and discuss situations in which it is possible to compute the optimal control law from these conditions.
\end{abstract}

\section{INTRODUCTION}

Two-dimensional (2-D) systems arise naturally in the modelling of processes where the variables are defined over space rather than time. Typical examples of latent variable models for linear shift-invariant 2-D systems are the Roesser [1] and Fornasini-Marchesini [2] models. Optimal control problems for Fornasini-Marchesini models are addressed, over an infinite frame, in [3] and [4]. The optimal control of 2-D systems over a finite frame is studied in [6], within the context of Roesser models. There, a variational approach is used to obtain necessary conditions for optimality. It was shown in [8] that the conditions presented in [6] are not only necessary, but also sufficient for the linear quadratic regulator (LQR) problem. Here we generalise those conditions to accomodate possible north-west affine constraints on the horizontal and vertical semi-states. This case can be considered as the two-dimensional counterpart of the problem formulated and solved in [5]. The solution of the equations which characterise the optimality condition is discussed, and a simple method to compute the optimal control law is described.

\section{PRoblem StATEMENT}

Consider the linear shift-invariant system described by the difference equation

$$
\left[\begin{array}{c}
h_{i+1, j} \\
v_{i, j+1}
\end{array}\right]=A\left[\begin{array}{c}
h_{i, j} \\
v_{i, j}
\end{array}\right]+B u_{i, j},
$$

in so-called Roesser form, where for all $i, j \in \mathbb{N}$, $h_{i, j} \in \mathbb{R}^{n_{h}}$ is the horizontal semi-state, $v_{i, j} \in \mathbb{R}^{n_{v}}$ is the vertical semi-state and $u_{i, j} \in \mathbb{R}^{m}$ is the control input. The matrices $A \in \mathbb{R}^{\left(n_{h}+n_{v}\right) \times\left(n_{h}+n_{v}\right)}$ and $B \in \mathbb{R}^{\left(n_{h}+n_{v}\right) \times m}$

Lorenzo Ntogramatzidis is with the Department of Mathematics and Statistics, Curtin University of Technology, Perth WA 6845, Australia L.Ntogramatzidis@curtin.edu.au

Michael Cantoni is with the Department of Electrical and Electronic Engineering, The University of Melbourne, Parkville VIC 3010, Australia cantoni@unimelb.edu.au are partitioned comformably with the semi-states $h_{i, j}$ and $v_{i, j} ;$ in particular,

$$
A=\left[\begin{array}{ll}
A_{1,1} & A_{1,2} \\
A_{2,1} & A_{2,2}
\end{array}\right] \text { and } B=\left[\begin{array}{c}
B_{1} \\
B_{2}
\end{array}\right] .
$$

Consider the bounded frame $\mathcal{Q}:=[0, N] \times[0, M]$, and notice that if we assign the input $u_{i, j}$ on $\mathcal{Q}$, along with the boundary conditions

$$
\begin{gathered}
h_{0, j}=a_{j} \in \mathbb{R}^{n_{h}} \quad j \in[0, M], \\
v_{i, 0}=b_{i} \in \mathbb{R}^{n_{v}} \quad i \in[0, N],
\end{gathered}
$$

then (1) uniquely determines the value of the horizontal semi-state over the bounded-frame $\mathcal{H}:=[0, N+1] \times$ $[0, M]$ and the value of the vertical semi-state over $\mathcal{V}:=$ $[0, N] \times[0, M+1]$.

Let $p_{j} \in\left\{0, \ldots, n_{h}\right\}$ and $W_{j} \in \mathbb{R}^{p_{j} \times n_{h}}$ be full rowrank for $j \in[0, M]$; let also $q_{i} \in\left\{0, \ldots, n_{v}\right\}$ and $Z_{i} \in$ $\mathbb{R}^{q_{i} \times n_{v}}$ be full row-rank for $i \in[0, N]$. Let $w_{j} \in \mathbb{R}^{p_{j}}$ and $z_{i} \in \mathbb{R}^{q_{i}}$ for all $j \in[0, M]$ and $i \in[0, N]$. Consider the constraints on the horizontal semistate on the region $\{N+1\} \times[0, M] \subset \mathbb{N} \times \mathbb{N}$ given by

$$
W_{j} h_{N+1, j}=w_{j} \quad \text { for } \quad j \in[0, M],
$$

and the constraints on the vertical semistate on $[0, M] \times$ $\{M+1\} \subset \mathbb{N} \times \mathbb{N}$ expressed by

$$
Z_{i} v_{i, M+1}=z_{i} \quad \text { for } \quad i \in[0, N] .
$$

For the sake of conciseness, these constraints will be referred to as 'north-west' constraints. If $p_{j}=0$ for a given $j \in[0, M]$, matrix $W_{j}$ has zero rows, and the vector $w_{j}$ has zero entries. In this situation, the constraint represented by (4) disappears, i.e., all the components of the vector $h_{N+1, j}$ are unconstrained. Likewise, if $q_{i}=$ 0 for $i \in[0, N]$, then $Z_{i}$ has zero rows, and $z_{i}$ has zero entries, so that all the components of $v_{i, M+1}$ are unconstrained. Let

$$
\begin{aligned}
J: & =\mathbf{h}_{N+1}^{\top} H \mathbf{h}_{N+1}+\mathbf{v}_{M+1}^{\top} V \mathbf{v}_{M+1} \\
& +\sum_{(i, j) \in \mathcal{Q}}\left[\begin{array}{lll}
h_{i, j}^{\top} & v_{i, j}^{\top} & u_{i, j}^{\top}
\end{array}\right] \Pi\left[\begin{array}{c}
h_{i, j} \\
v_{i, j} \\
u_{i, j}
\end{array}\right]
\end{aligned}
$$

be the performance index to minimise, where $\mathbf{h}_{N+1}:=\left[\begin{array}{lll}h_{N+1,0}^{\top} & \ldots & h_{N+1, M}^{\top}\end{array}\right]^{\top}, \mathbf{v}_{M+1}:=$ $\left[\begin{array}{lll}v_{0, M+1}^{\top} & \cdots & v_{N, M+1}^{\top}\end{array}\right]^{\top}$, and the symmetric and positive 
semidefinite matrices $H \in \mathbb{R}^{\left[n_{h}(M+1)\right] \times\left[n_{h}(M+1)\right]}$ and $V \in \mathbb{R}^{\left[n_{v}(N+1)\right] \times\left[n_{v}(N+1)\right]}$ penalise the (north-east) boundary values of the horizontal and vertical semi-states, respectively. The so-called Popov matrix $\Pi$ appearing in (6) is partitioned as

$$
\Pi=\left[\begin{array}{cc}
Q & S \\
S^{\top} & R
\end{array}\right]=\Pi^{\top} \geq 0,
$$

and the matrices $Q \in \mathbb{R}^{\left(n_{h}+n_{v}\right) \times\left(n_{h}+n_{v}\right)}$ and $S \in$ $\mathbb{R}^{\left(n_{h}+n_{v}\right) \times m}$ are partitioned conformably with the semistates $h_{i, j}$ and $v_{i, j}$, respectively; in particular,

$$
Q=\left[\begin{array}{ll}
Q_{1,1} & Q_{1,2} \\
Q_{1,2}^{\top} & Q_{2,2}
\end{array}\right] \quad \text { and } \quad S=\left[\begin{array}{c}
S_{1} \\
S_{2}
\end{array}\right],
$$

where $Q_{1,1} \in \mathbb{R}^{n_{h} \times n_{h}}, Q_{1,2} \in \mathbb{R}^{n_{v} \times n_{v}}, Q_{2,2} \in \mathbb{R}^{n_{v} \times n_{v}}$, $S_{1} \in \mathbb{R}^{n_{h} \times m}$ and $S_{2} \in \mathbb{R}^{n_{v} \times m}$.

Note that it is not assumed that $R$ is non-singular at this stage. It is now possible to formally define the optimal control problem considered in this paper as follows.

Problem 1: Find the control input $u_{i, j}$, for $(i, j) \in \mathcal{Q}$, minimising the quadratic performance index (6) subject to (1), (3), (4) and (5).

The formulation of Problem 1 involves an affine constraint on the semistates $h_{N+1, j}$ and $v_{i, M+1}$ for $j \in[0, M]$ and $i \in[0, N]$. As such, this formulation encompasses the standard case where $h_{N+1, j}$ and $v_{i, M+1}$ are not constrained (by setting $p_{j}=0$ for all $j \in[0, M]$ and $q_{i}=0$ for all $i \in[0, N]$ ). It also covers the so-called 'double-ended boundary value problem', in which all components of the vectors $h_{N+1, j}$ and $v_{i, M+1}$ are fixed for all $j \in[0, M]$ and $i \in[0, N]$, by setting $p_{j}=n_{h}$ for all $j \in[0, M]$ and $q_{i}=n_{v}$ for all $i \in[0, N]$. In this case, the matrices $H$ and $V$ in the performance index (6) are set to zero, as the quadratic penalisation on the vectors $h_{N+1, j}$ and $v_{i, M+1}$ leads to a constant offset in the cost.

\section{Solution of Problem 1}

In this section, three important points related to an open-loop solution to Problem 1 are addressed. First, we present a generalised version of the optimality conditions established in [6] in order to accomodate the northwest affine constraints (4-5). Note that the conditions presented here are not only necessary, but also sufficient for optimality. It is then shown how the optimal control can be explicitly computed from these conditions, under a set of simple assumptions.

\section{A. Necessary and sufficient conditions for optimality}

In [6], necessary optimality conditions for Problem 1 in standard form - i.e., without the north-west constraints considered here - are obtained as a special case of a condition established therein for a non-linear version of the constraint (1), via a variational analysis of the Lagrangian associated with (6). The question of sufficiency was resolved in [8]. Below, the optimality conditions are derived for the more general case of north-west constraints on the horizontal and vertical semi-states as defined in Problem 1. In the following theorem, the matrices $H$ and $V$ are considered to be partitioned into block submatrices of dimensions $n_{h} \times n_{h}$ and $n_{v} \times n_{v}$, respectively. These submatrices are denoted with the symbols $H_{i, j}(i, j \in$ $[0, M])$ and $V_{i, j}(i, j \in[0, N])$.

Theorem 1: If $u_{i, j}$, and the corresponding $h_{i, j}$ and $v_{i, j}$ are optimal for Problem 1, then there exist $\lambda_{i, j} \in \mathbb{R}^{n_{h}}$ for $(i, j) \in \mathcal{H}, \mu_{i, j} \in \mathbb{R}^{n_{v}}$ for $(i, j) \in \mathcal{V}, \xi_{j} \in \mathbb{R}^{p_{j}}$ for $j \in$ $[0, M]$ and $\zeta_{i} \in \mathbb{R}^{q_{i}}$ for $i \in[0, N]$ such that:

i) for all $j \in[0, M]$

$$
\begin{aligned}
h_{0, j} & =a_{j} \quad \text { and } \quad W_{j} h_{N+1, j}=w_{j}, \\
\lambda_{N+1, j} & =\sum_{k=0}^{M} H_{j, k} h_{N+1, j}+W_{j}^{\top} \xi_{j}
\end{aligned}
$$

ii) for all $i \in[0, N]$

$$
\begin{aligned}
v_{i, 0} & =b_{i} \quad \text { and } \quad Z_{i} v_{i, M+1}=z_{i}, \\
\mu_{i, M+1} & =\sum_{l=0}^{N} V_{l, i} v_{i, M+1}+Z_{i}^{\top} \zeta_{i} ;
\end{aligned}
$$

iii) for all $(i, j) \in \mathcal{Q}$

$$
\begin{aligned}
{\left[\begin{array}{l}
\lambda_{i, j} \\
\mu_{i, j}
\end{array}\right] } & =Q\left[\begin{array}{l}
h_{i, j} \\
v_{i, j}
\end{array}\right]+S u_{i, j}+A^{\top}\left[\begin{array}{l}
\lambda_{i+1, j} \\
\mu_{i, j+1}
\end{array}\right] \\
0 & =R u_{i, j}+S^{\top}\left[\begin{array}{l}
h_{i, j} \\
v_{i, j}
\end{array}\right]+B^{\top}\left[\begin{array}{l}
\lambda_{i+1, j} \\
\mu_{i, j+1}
\end{array}\right], \\
{\left[\begin{array}{c}
h_{i+1, j} \\
v_{i, j+1}
\end{array}\right] } & =A\left[\begin{array}{l}
h_{i, j} \\
v_{i, j}
\end{array}\right]+B u_{i, j} .
\end{aligned}
$$

Conversely, if (7-13) admit solutions $h_{i, j}, v_{i, j}, \lambda_{i, j}, \mu_{i, j}$, $u_{i, j}, \xi_{j}$ and $\zeta_{i}$, then the corresponding $h_{i, j}, v_{i, j}$ and $u_{i, j}$ minimize $J$ subject to the constraints (1), (3), (4-5).

The proof of this theorem can be carried out along the same lines of that in [8].

Remark 1: Similarly to the 1-D case, any constraint on components of the semi-state vector correspond to components of the semi-costate vector that are unconstrained (and vice-versa). In fact, it is easy to see that, due to the constraint $W_{j} h_{N+1, j}=w_{j}$, the components of the corresponding semi-costate vector $\lambda_{N+1, j}$ are constrained on the subspace $\operatorname{ker} W_{j}$. The freedom of the remaining components is represented by the components of the vector $\xi_{j}$. In particular:

- when all the components of the semi-state $h_{N+1, j}$ are unconstrained, i.e., when $p_{j}=0$, we can assume $\operatorname{ker} W_{j}=\mathbb{R}^{n_{h}}$, which implies that all the components of the semi-costate $\lambda_{N+1, j}$ are constrained, and $\xi_{j}$ disappears (or, more precisely, it is a vector of $p_{j}=0$ entries);

- when all the components of the semi-state $h_{N+1, j}$ are constrained, i.e., when $p_{j}=n_{h}$, then $\operatorname{ker} W_{j}=\{0\}$ since $W_{j}$ is invertible, and $\xi_{j}$ is an $n_{h}$-dimensional vector. It follows that there are no constraints on 
$\lambda_{N+1, j}$, since (8) gives rise to $n_{h}$ equations, but the number of free variables equals the number of components of $\xi_{j}$, i.e., $n_{h}$.

The considerations above can obviously apply to the vertical semi-state with minor modifications.

Remark 2: Let us consider the case where $p_{j}=0$ for all $j \in[0, M]$ and $q_{i}=0$ for all $i \in[0, N]$, i.e., where there are no constraints on the semistates $h_{N+1, j}$ and $v_{i, M+1}$ for $j \in[0, M]$ and $i \in[0, N]$. Since in this case any control input to (1) is admissible for the specified boundary conditions (3), in the sense that a corresponding semi-state trajectory always exists, Problem 1 can be re-expressed as a feasible static quadratic optimisation problem in the $l=m[(N+1) \cdot(M+1)]$ unconstrained variables representing the control input on $\mathcal{Q}$. In particular, Problem 1 always admits solutions in this case. Thus, by virtue of Theorem 1, the 'double-ended boundary value problem' in the semi-state and semi-costate vectors (713) always admits solutions. In the next sections, the computation of such solutions is discussed in more detail. When the constraints (4-5) are imposed, on the other hand, Problem 1 admits solutions if and only if there exists a trajectory generated by (1) that satisfies (3-5).

\section{B. Solving the double-ended boundary value problem}

As aforementioned, (7-13) constitute a two-dimensional 'double-ended boundary value problem' in the semi-state and semi-costate vectors. In general, this problem is difficult to solve. However, in several situations of interest an explicit solution can be obtained. In this section it is shown that, under some assumption on the problem matrices, equations (11), (12) and (13) can be re-expressed as a standard Roesser model. The set of solutions can be written in a parameterised form, using the transition matrix recursion given in [1]. Then, it is shown how to impose the boundary conditions (8) and (10) to determine the parameters. This leads to a simple linear equation for the parameters, which must admit solutions in order for the optimal control problem to be feasible. When a solution for the parameters exists, the corresponding input and semi-state functions are optimal by construction, as they satisfy all the necessary and sufficient conditions in Theorem 1 .

When $R$ is positive definite (and hence invertible), equation (12) can be solved for $u_{i, j}$, so that (11) and (13) can be written together as

$$
\begin{gathered}
{\left[\begin{array}{cc}
I & B R^{-1} B^{\top} \\
0 & A^{\top}-S R^{-1} B^{\top}
\end{array}\right]\left[\begin{array}{c}
h_{i+1, j} \\
v_{i, j+1} \\
\lambda_{i+1, j} \\
\mu_{i, j+1}
\end{array}\right]=} \\
{\left[\begin{array}{cc}
A-B R^{-1} S^{\top} & 0 \\
S R^{-1} S^{\top}-Q & I
\end{array}\right]\left[\begin{array}{c}
h_{i, j} \\
v_{i, j} \\
\lambda_{i, j} \\
\mu_{i, j}
\end{array}\right] .}
\end{gathered}
$$

The matrices appearing above are invertible iff $A-$ $B R^{-1} S^{\top}$ is invertible. In this case, (14) can be rewritten as

$$
\left[\begin{array}{c}
r_{i+1, j} \\
s_{i, j+1}
\end{array}\right]=\left[\begin{array}{ll}
P_{1} & P_{2} \\
P_{3} & P_{4}
\end{array}\right]\left[\begin{array}{c}
r_{i, j} \\
s_{i, j}
\end{array}\right],
$$

where $\left[\begin{array}{ll}P_{1} & P_{2} \\ P_{3} & P_{4}\end{array}\right]:=\left[\begin{array}{ll}G_{1,1}^{\prime} & G_{1,2}^{\prime \prime} \\ G_{2,1}^{\prime \prime} & G_{2,2}^{\prime}\end{array}\right]^{-1}\left[\begin{array}{ll}T_{1,1}^{\prime} & T_{1,2}^{\prime \prime} \\ T_{2,1}^{\prime \prime} & T_{2,2}^{\prime}\end{array}\right]$, with

$$
\begin{aligned}
G_{k, l}^{\prime} & :=\left[\begin{array}{cc}
I & B_{k} R^{-1} B_{l}^{\top} \\
0 & A_{k, l}^{\top}-S_{k} R^{-1} B_{l}^{\top}
\end{array}\right], \\
G_{k, l}^{\prime \prime} & :=\left[\begin{array}{cc}
0 & B_{k} R^{-1} B_{l}^{\top} \\
0 & A_{k, l}^{\top}-S_{k} R^{-1} B_{l}^{\top}
\end{array}\right], \\
T_{k, l}^{\prime} & :=\left[\begin{array}{cc}
A_{k, l}-B_{k} R^{-1} S_{l}^{\top} & 0 \\
S_{k} R^{-1} S_{l}^{\top}-Q_{k, l} & I
\end{array}\right], \\
T_{k, l}^{\prime \prime} & :=\left[\begin{array}{ll}
A_{k, l}-B_{k} R^{-1} S_{l}^{\top} & 0 \\
S_{k} R^{-1} S_{l}^{\top}-Q_{k, l} & 0
\end{array}\right],
\end{aligned}
$$

and $r_{i, j}:=\left[\begin{array}{l}h_{i, j} \\ \lambda_{i, j}\end{array}\right]$ and $s_{i, j}:=\left[\begin{array}{l}v_{i, j} \\ \mu_{i, j}\end{array}\right]$. Model (15) is in Roesser form. Therefore, the semi-state transition matrix associated with (15) can be computed by using the general response formula given in [1]:

$$
\begin{aligned}
& \Phi_{0,0}=I \\
& \Phi_{i, j}=0 \quad \text { if } i<0 \text { or } j<0 ; \\
& \Phi_{i, j}=\Phi_{1,0} \Phi_{i-1, j}+\Phi_{0,1} \Phi_{i, j-1} \quad \forall(i, j) \in \mathbb{N} \backslash\{(0,0)\},
\end{aligned}
$$

where $\Phi_{1,0}:=\left[\begin{array}{cc}P_{1} & P_{2} \\ 0 & 0\end{array}\right]$ and $\Phi_{0,1}:=\left[\begin{array}{cc}0 & 0 \\ P_{3} & P_{4}\end{array}\right]$. Now, if we consider the matrix $\Phi_{i, j}$ to be partitioned as $\Phi_{i, j}=$ $\left[\begin{array}{c}\Phi_{i, j}^{1} \Phi_{i, j}^{2} \\ \Phi_{i, j}^{3} \Phi_{i, j}^{4}\end{array}\right]$ conformably with $\left[\begin{array}{l}r_{i, j} \\ s_{i, j}\end{array}\right]$, where each $\Phi_{i, j}^{k}$, for $k \in\{1,2,3,4\}$, is in turn partitioned as $\Phi_{i, j}^{k}=$ $\left[\begin{array}{ll}\Phi_{i, j}^{k, 1} & \Phi_{i, j}^{k, 2} \\ \Phi_{i, j}^{k, 3} & \Phi_{i, j}^{k, 4}\end{array}\right]$, conformably with $\left[\begin{array}{l}h_{i, j} \\ \lambda_{i, j}\end{array}\right]$ and $\left[\begin{array}{l}v_{i, j} \\ \mu_{i, j}\end{array}\right]$, we can write the solution of (15) explicitly as

$$
\begin{aligned}
h_{i, j}= & \sum_{k=0}^{j}\left(\Phi_{i, j-k}^{1,1} a_{k}+\Phi_{i, j-k}^{1,2} \lambda_{0, k}\right) \\
& +\sum_{h=0}^{i}\left(\Phi_{i-h, j}^{2,1} b_{h}+\Phi_{i-h, j}^{2,2} \mu_{h, 0}\right), \\
\lambda_{i, j}= & \sum_{k=0}^{j}\left(\Phi_{i, j-k}^{1,3} a_{k}+\Phi_{i, j-k}^{1,4} \lambda_{0, k}\right) \\
& +\sum_{h=0}^{i}\left(\Phi_{i-h, j}^{2,3} b_{h}+\Phi_{i-h, j}^{2,4} \mu_{h, 0}\right), \\
v_{i, j}= & \sum_{k=0}^{j}\left(\Phi_{i, j-k}^{3,1} a_{k}+\Phi_{i, j-k}^{3,2} \lambda_{0, k}\right) \\
& +\sum_{h=0}^{i}\left(\Phi_{i-h, j}^{4,1} b_{h}+\Phi_{i-h, j}^{4,2} \mu_{h, 0}\right),
\end{aligned}
$$




$$
\begin{aligned}
\mu_{i, j}= & \sum_{k=0}^{j}\left(\Phi_{i, j-k}^{3,3} a_{k}+\Phi_{i, j-k}^{3,4} \lambda_{0, k}\right) \\
& +\sum_{h=0}^{i}\left(\Phi_{i-h, j}^{4,3} b_{h}+\Phi_{i-h, j}^{4,4} \mu_{h, 0}\right),
\end{aligned}
$$

where the boundary conditions (3) have been used. From these semi-state trajectories satisfying (3), we now need to select those that also satisfy the remaining equations in Theorem 1. For the sake of simplifying the notation, suppose that matrices $H$ and $V$ are diagonal, i.e., $H=$ $\operatorname{diag}\left\{H_{0,0}, H_{1,1}, \ldots, H_{M, M}\right\}$, where $H_{j, j} \in \mathbb{R}^{n_{h}}$ for all $j \in\{0, \ldots, M\}$, and $V=\operatorname{diag}\left\{V_{0,0}, V_{1,1}, \ldots, V_{N, N}\right\}$, where $V_{i, i} \in \mathbb{R}^{n_{v}}$ for all $i \in\{0, \ldots, N\}$. Imposing (8) on (16) and (17) gives

$$
\begin{aligned}
\lambda_{N+1, j} & =\sum_{k=0}^{j}\left(\Phi_{N+1, j-k}^{1,3} a_{k}+\Phi_{N+1, j-k}^{1,4} \lambda_{0, k}\right) \\
& +\sum_{h=0}^{N}\left(\Phi_{N+1-h, j}^{2,3} b_{h}+\Phi_{N+1-h, j}^{2,4} \mu_{h, 0}\right) \\
& =H_{j, j}\left(\sum_{k=0}^{j}\left(\Phi_{N+1, j-k}^{1,1} a_{k}+\Phi_{N+1, j-k}^{1,2} \lambda_{0, k}\right)\right. \\
& \left.+\sum_{h=0}^{N}\left(\Phi_{N+1-h, j}^{2,1} b_{h}+\Phi_{N+1-h, j}^{2,2} \mu_{h, 0}\right)\right)+W_{j}^{\top} \xi_{j},
\end{aligned}
$$

while imposing $W_{j} h_{N+1, j}=w_{j}$ on (16) gives

$$
\begin{aligned}
W_{j} & {\left[\sum_{k=0}^{j}\left(\Phi_{N+1, j-k}^{2,1} a_{k}+\Phi_{N+1, j-k}^{1,2} \lambda_{0, k}\right)\right.} \\
& \left.+\sum_{h=0}^{N+1}\left(\Phi_{N+1-h, j}^{2,1} b_{h}+\Phi_{N+1-h, j}^{2,2} \mu_{h, 0}\right)\right]=w_{j},
\end{aligned}
$$

which represents $p_{j}$ equations in the $\left[(M+1) n_{h}\right] \times$ $\left[(N+1) n_{v}\right]$ unknowns $\lambda_{0,0}, \ldots, \lambda_{0, M}, \mu_{0,0}, \ldots, \mu_{N, 0}$. Let $j \in[0, M]$ be such that the full row-rank matrix $W_{j}$ is not square, i.e., $\operatorname{ker} W_{j} \neq\{0\}$, and let $\tilde{W}_{j}$ be a basis matrix for the null-space of $W_{j}$, so that $\tilde{W}_{j}^{\top} W_{j}^{\top}=0$; By premultiplying (20) by $\tilde{W}_{j}^{\top}$ we obtain

$$
\begin{aligned}
& \tilde{W}_{j}^{\top}\left[\sum_{k=0}^{j}\left(\Phi_{N+1, j-k}^{1,4}-H_{j, j} \Phi_{N+1, j-k}^{1,2}\right) \lambda_{0, k}\right. \\
& \left.+\sum_{h=0}^{N}\left(\Phi_{N+1-h, j}^{2,4}-H_{j, j} \Phi_{N+1-h, j}^{2,2}\right) \mu_{h, 0}\right]= \\
& -\tilde{W}_{j}^{\top}\left[\sum_{k=0}^{j}\left(\Phi_{N+1, j-k}^{1,3}-H_{j, j} \Phi_{N+1, j-k}^{1,1}\right) a_{k}+\right. \\
& \left.+\sum_{h=0}^{N}\left(\Phi_{N+1-h, j}^{2,3}-H_{j, j} \Phi_{N+1-h, j}^{2,1}\right) b_{h}\right] .
\end{aligned}
$$

As before, (22) represents $n_{h}-p_{j}$ equations in the $\left[(M+1) n_{h}\right] \times\left[(N+1) n_{v}\right]$ unknowns $\lambda_{0,0}, \ldots, \lambda_{0, M}, \mu_{0,0}, \ldots, \mu_{N, 0}$. Similarly, (10) and (18-19) yield

$$
\begin{aligned}
& \mu_{i, M+1}=\sum_{k=0}^{M}\left(\Phi_{i, M+1-k}^{3,3} a_{k}+\Phi_{i, M+1-k}^{3,4} \lambda_{0, k}\right) \\
& +\sum_{h=0}^{i}\left(\Phi_{i-h, M+1}^{4,3} b_{h}+\Phi_{i-h, M+1}^{4,4} \mu_{h, 0}\right) \\
& =V_{i, i}\left(\sum_{k=0}^{M}\left(\Phi_{i, M+1-k}^{3,1} a_{k}+\Phi_{i, M+1-k}^{3,2} \lambda_{0, k}\right)\right. \\
& \left.+\sum_{h=0}^{i}\left(\Phi_{i-h, M+1}^{4,1} b_{h}+\Phi_{i-h, M+1}^{4,2} \mu_{h, 0}\right)\right)+Z_{i}^{\top} \zeta_{i},
\end{aligned}
$$

while imposing $Z_{i} v_{i, M+1}=z_{i}$ on (18) gives

$$
\begin{aligned}
Z_{i}\left[\sum_{k=0}^{M}\left(\Phi_{i, M+1-k}^{3,2} \lambda_{0, k}+\Phi_{i, M+1-k}^{3,1} a_{k}\right)\right. & \\
& \left.+\sum_{h=0}^{i}\left(\Phi_{i-h, M+1}^{4,2} \mu_{h, 0}+\Phi_{i-h, M+1}^{4,1} b_{h}\right)\right]=z_{i}
\end{aligned}
$$

Let $i \in[0, N]$ be such that $Z_{i}$ is not square, i.e., $\operatorname{ker} Z_{i} \neq$ $\{0\}$, and let $\tilde{Z}_{i}$ be, for all $i \in[0, N]$, a basis matrix for the null-space of $Z_{i}$, so that $\tilde{Z}_{i}^{\top} Z_{i}^{\top}=0$. By premultiplying (23) by $\tilde{Z}_{i}^{\top}$, we get

$$
\begin{aligned}
& \tilde{Z}_{i}^{\top}\left[\sum_{k=0}^{M}\left(\Phi_{i, M+1-k}^{3,4}-V_{i, i} \Phi_{i, M+1-k}^{3,2}\right) \lambda_{0, k}\right. \\
& \left.+\sum_{h=0}^{i}\left(\Phi_{i, M+1-k}^{4,4}-V_{i, i} \Phi_{i, M+1-k}^{4,2}\right) \mu_{h, 0}\right]= \\
& -\tilde{Z}_{i}^{\top}\left[\sum_{k=0}^{M}\left(\Phi_{i, M+1-k}^{3,3}-V_{i, i} \Phi_{i, M+1-k}^{3,1}\right) a_{k}\right. \\
& \left.+\sum_{h=0}^{i}\left(\Phi_{i, M+1-k}^{4,3}-V_{i, i} \Phi_{i, M+1-k}^{4,1}\right) b_{h}\right] .
\end{aligned}
$$

Equations (21), (22), (24) and (25) can be expressed in 
the following compact linear form

$$
\begin{aligned}
{\left[\begin{array}{c|c}
\Psi_{\lambda} & \Psi_{\mu} \\
\hline \Xi_{\lambda} & \Xi_{\mu}
\end{array}\right]\left[\begin{array}{c}
\lambda_{0,0} \\
\vdots \\
\frac{\lambda_{0, M}}{\mu_{0,0}} \\
\vdots \\
\mu_{N, 0}
\end{array}\right]=} \\
-\left[\begin{array}{c|c}
\Theta_{a} & \Theta_{b} \\
\hline \Gamma_{a} & \Gamma_{b}
\end{array}\right]\left[\begin{array}{c}
a_{0} \\
\vdots \\
a_{M} \\
b_{0} \\
\vdots \\
b_{N}
\end{array}\right]+\left[\begin{array}{c}
\hat{w}_{0} \\
\vdots \\
\hat{w}_{M} \\
\hat{z}_{0} \\
\vdots \\
\hat{z}_{N}
\end{array}\right],
\end{aligned}
$$

where the matrices $\Omega_{i, j}$, for $i, j \in\{1,2\}$, are given by

$$
\begin{gathered}
\Psi_{\lambda}:=\left[\begin{array}{cccc}
\Psi_{N+1,0,0}^{1} & 0 & \ldots & 0 \\
\Psi_{N+1,1,1}^{1} & \Psi_{N+1,0,1}^{1} & \ldots & 0 \\
\vdots & \vdots & \ddots & \vdots \\
\Psi_{N+1, M, M}^{1} & \Psi_{N+1, M-1, M}^{1} & \ldots & \Psi_{N+1,0, M}^{1}
\end{array}\right], \\
\Psi_{\mu}:=\left[\begin{array}{cccc}
\Psi_{N+1,0,0}^{2} & \Psi_{N, 0,0}^{2} & \ldots & \Psi_{1,0,0}^{2} \\
\Psi_{N+1,1,1}^{2} & \Psi_{N, 1,1}^{2} & \ldots & \Psi_{1,1,1}^{2} \\
\vdots & \vdots & \ddots & \vdots \\
\Psi_{N+1, M, M}^{2} & \Psi_{N, M, M}^{2} & \ldots & \Psi_{1, M, M}^{2}
\end{array}\right], \\
\Xi_{\lambda}:=\left[\begin{array}{cccc}
\Xi_{0, M+1,0}^{3} & \Xi_{0, M, 0}^{3} & \ldots & \Xi_{0,1,0}^{3} \\
\Xi_{1, M+1,1}^{3} & \Xi_{1, M, 1}^{3} & \ldots & \Xi_{1,1,1}^{3} \\
\vdots & \vdots & \ddots & \vdots \\
\Xi_{N, M+1, M}^{3} & \Xi_{N, M, N}^{3} & \ldots & \Xi_{N, 1, N}^{3}
\end{array}\right], \\
\Xi_{\mu}:=\left[\begin{array}{cccc}
\Xi_{0, M+1,0}^{4} & 0 & \ldots & 0 \\
\Xi_{1, M+1,1}^{4} & \Xi_{0, M+1,1}^{4} & \ldots & 0 \\
\vdots & \vdots & \ddots & \vdots \\
\Xi_{N, M+1, N}^{4} & \Xi_{N-1, M+1, N}^{4} & \ldots & \Xi_{0, M+1, N}^{4}
\end{array}\right],
\end{gathered}
$$

and the matrices $\Upsilon_{i, j}$, for $i, j \in\{1,2\}$, are given by

$$
\begin{gathered}
\Theta_{a}:=\left[\begin{array}{cccc}
\Theta_{N+1,0,0}^{1} & 0 & \ldots & 0 \\
\Theta_{N+1,0,1}^{1} & \Theta_{N+1,1,1}^{1} & \ldots & 0 \\
\vdots & \vdots & \ddots & \vdots \\
\Theta_{N+1, M, M}^{1} & \Theta_{N+1, M-1, M}^{1} & \ldots & \Theta_{N+1,0, M}^{1}
\end{array}\right], \\
\Theta_{b}:=\left[\begin{array}{cccc}
\Theta_{N+1,0,0}^{2} & \Theta_{N, 0,0}^{2} & \ldots & \Theta_{1,0,0}^{2} \\
\Theta_{N+1,1,1}^{2} & \Theta_{N, 1,1}^{2} & \ldots & \Theta_{1,1,1}^{2} \\
\vdots & \vdots & \ddots & \vdots \\
\Theta_{N+1, M, M}^{2} & \Theta_{N, M, M}^{2} & \ldots & \Theta_{1, M, M}^{2}
\end{array}\right], \\
\Gamma_{a}:=\left[\begin{array}{cccc}
\Gamma_{0, M+1,0}^{3} & \Gamma_{0, M, 0}^{3} & \ldots & \Gamma_{0,1,0}^{3} \\
\Gamma_{1, M+1,1}^{3} & \Gamma_{1, M, 1}^{3} & \ldots & \Gamma_{1,1,1}^{3} \\
\vdots & \vdots & \ddots & \vdots \\
\Gamma_{N, M+1, N}^{3} & \Gamma_{N, M, N}^{3} & \ldots & \Gamma_{N, 1, N}^{3}
\end{array}\right],
\end{gathered}
$$

$$
\Gamma_{b}:=\left[\begin{array}{cccc}
\Gamma_{0, M+1,0}^{4} & 0 & \cdots & 0 \\
\Gamma_{1, M+1,1}^{4} & \Gamma_{0, M+1,1}^{4} & \cdots & 0 \\
\vdots & \vdots & \ddots & \vdots \\
\Gamma_{N, M+1, N}^{4} & \Gamma_{N-1, M+1, N}^{4} & \cdots & \Gamma_{0, M+1, N}^{4}
\end{array}\right],
$$

where

$$
\begin{aligned}
& \Psi_{h, l, k}^{X}:= {\left[\begin{array}{c}
\tilde{W}_{k}^{\top}\left(\Phi_{h, l}^{X, 4}-H_{k, k} \Phi_{h, l}^{X, 2}\right) \\
W_{k} \Phi_{h, l}^{X, 2}
\end{array}\right], } \\
& \Theta_{h, l, k}^{X}:=\left[\begin{array}{c}
\tilde{W}_{k}^{\top}\left(\Phi_{h, l}^{X, 3}-H_{k, k} \Phi_{h, l}^{X, 1}\right) \\
W_{k} \Phi_{h, l}^{X, 1}
\end{array}\right], \\
& \Xi_{h, l, k}^{X}:=\left[\begin{array}{c}
\tilde{Z}_{k}^{\top}\left(\Phi_{h, l}^{X, 4}-V_{k, k} \Phi_{h, l}^{X, 2}\right) \\
Z_{k} \Phi_{h, l}^{X, 2}
\end{array}\right], \\
& \Gamma_{h, l, k}^{X}:=\left[\begin{array}{c}
\tilde{Z}_{k}^{\top}\left(\Phi_{h, l}^{X, 3}-V_{k, k} \Phi_{h, l}^{X, 1}\right) \\
Z_{k} \Phi_{h, l}^{X, 1}
\end{array}\right],
\end{aligned}
$$

with $X \in\{1,2,3,4\}$, and where

$$
\hat{w}_{j}:=\left[\begin{array}{c}
0_{n_{h}-p_{j}} \\
w_{j}
\end{array}\right] \quad \text { and } \quad \hat{z}_{i}:=\left[\begin{array}{c}
0_{n_{v}-q_{i}} \\
z_{i}
\end{array}\right] .
$$

All matrices $\Psi_{i, j}^{h, l, k}$ and $\Theta_{i, j}^{h, l, k}$ have $n_{h}$ rows, while all matrices $\Xi_{i, j}^{h, l, k}$ and $\Gamma_{i, j}^{h, l, k}$ have $n_{v}$ rows. In fact, $W_{j}$ has $p_{j}$ rows and matrix $\tilde{W}_{j}^{\top}$ has $n_{h}-p_{j}$ rows, while $Z_{i}$ has $q_{i}$ rows and matrix $\tilde{Z}_{i}^{\top}$ has $n_{v}-q_{i}$ rows. It follows that $\Omega_{1,1}$ and $\Upsilon_{1,1}$ are $\left[(M+1) n_{h}\right] \times\left[(M+1) n_{h}\right], \Omega_{1,2}$ and $\Upsilon_{1,2}$ are $\left[(M+1) n_{h}\right] \times\left[(N+1) n_{h}\right], \Omega_{2,1}$ and $\Upsilon_{2,1}$ are $\left[(N+1) n_{v}\right] \times\left[(M+1) n_{v}\right], \Omega_{2,2}$ and $\Upsilon_{2,2}$ are $[(N+$ 1) $\left.n_{v}\right] \times\left[(N+1) n_{v}\right]$. As such, $(26)$ consists of $[(M+$ 1) $\left.n_{h}\right] \times\left[(N+1) n_{v}\right]$ equations in the $\left[(M+1) n_{h}\right] \times$ $\left[(N+1) n_{v}\right]$ unknowns $\lambda_{0,0}, \ldots, \lambda_{0, M}, \mu_{0,0}, \ldots, \mu_{N, 0}$.

In view of Remark 2 , when $p_{j}=0$ for some $j \in[0, M]$, we can take $\tilde{W}_{j}=I_{n_{h}}$. In this case, equation (21) disappears, and the vector $w_{j}$ has zero entries. Similarly, when $q_{i}=0$ for some $i \in[0, N]$, we can take $\tilde{Z}_{i}=I_{n_{v}}$, so that (24) disappears, and $z_{j}$ has zero entries. In this case, the boundary value problem always admits solutions, and as such, given arbitrary choice of the boundary condition parameters $a_{i}$ and $b_{j}$, the linear equation (26) admits solutions; i.e. im $\left[\begin{array}{ll}\Upsilon_{1,1} & \Upsilon_{1,2} \\ \Upsilon_{2,1} & \Upsilon_{2,2}\end{array}\right] \subseteq \operatorname{im}\left[\begin{array}{ll}\Omega_{1,1} & \Omega_{1,2} \\ \Omega_{2,1} & \Omega_{2,2}\end{array}\right]$, since $\hat{w}_{j}$ and $\hat{z}_{i}$ are all vectors with zero entries. Also note that in this case the solutions of (26) are parameterised in the

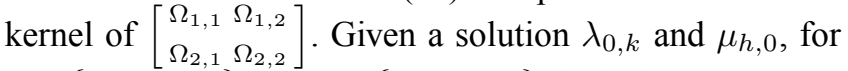
$k \in\{0, \ldots, M\}$ and $h \in\{0, \ldots, N\}$, both the semi-states $h_{i, j}$ and $v_{i, j}$ and the semi-costates $\lambda_{i, j}$ and $\mu_{i, j}$ can be determined for $(i, j) \in \mathcal{Q}$ by using (16-19). The semistate and semi-costate trajectories thus obtained satisfy the Roesser model (15) - which is equivalent to a linear combination of (11), (12) and (13) under the assumptions considered - and the boundary conditions (8), (10), (7) and (9). Finally, by using (12), the control $u_{i, j}$ can be computed for all $(i, j) \in \mathcal{Q}$, since $R$ is invertible by hypothesis here. The semi-state trajectories and the control 
obtained are therefore optimal, since they satisfy all the necessary and sufficient conditions for optimality; i.e., (713) given in Theorem 1.

If $p_{j}=n_{h}$ for some $j \in[0, M]$, or $q_{i}=n_{v}$ for some $i \in[0, N]$, the corresponding equations (20) or (23) can be neglected. In this case, the first block-row of matrices $\Psi_{h, l, k}^{X}, \Theta_{h, l, k}^{X}, \Xi_{h, l, k}^{X}$ and $\Gamma_{h, l, k}^{X}$ disappear.

Remark 3: Clearly, the assumption that $A-B R^{-1} S^{\top}$ be invertible causes some loss of generality. On the other hand, both $R$ and $S$ are weighting matrices that are usually chosen by the designer to obtain a satisfactory behaviour of the semi-states and of the control input. Hence, in most cases - with possible problems arising when the pair $(A, B)$ has uncontrollable eigenvalues at zero - matrices $R$ and $S$ can be chosen so as to meet the condition on $A-B R^{-1} S^{\top}$.

Remark 4: When the matrices $H$ and $V$ are not diagonal, the procedure outlined above still applies with little modifications. In this case, we find that equations (20) and (23) become respectively

$$
\begin{aligned}
& \lambda_{N+1, j}=\sum_{k=0}^{j}\left(\Phi_{N+1, j-k}^{1,3} a_{k}+\Phi_{N+1, j-k}^{1,4} \lambda_{0, k}\right) \\
&+\sum_{h=0}^{N}\left(\Phi_{N+1-h, j}^{2,3} b_{h}+\Phi_{N+1-h, j}^{2,4} \mu_{h, 0}\right) \\
&=\sum_{k=0}^{M} H_{j, k}\left(\sum_{k=0}^{j}\left(\Phi_{N+1, j-k}^{1,1} a_{k}+\Phi_{N+1, j-k}^{1,2} \lambda_{0, k}\right)\right. \\
&\left.+\sum_{h=0}^{N}\left(\Phi_{N+1-h, j}^{2,1} b_{h}+\Phi_{N+1-h, j}^{2,2} \mu_{h, 0}\right)\right)+W_{j}^{\top} \xi_{j}
\end{aligned}
$$

and

$$
\begin{aligned}
& \mu_{i, M+1}=\sum_{k=0}^{M}\left(\Phi_{i, M+1-k}^{3,3} a_{k}+\Phi_{i, M+1-k}^{3,4} \lambda_{0, k}\right) \\
&+\sum_{h=0}^{i}\left(\Phi_{i-h, M+1}^{4,3} b_{h}+\Phi_{i-h, M+1}^{4,4} \mu_{h, 0}\right) \\
&=\sum_{k=0}^{N} V_{k, i}\left(\sum_{k=0}^{M+1}\left(\Phi_{i, M+1-k}^{3,1} a_{k}+\Phi_{i, M+1-k}^{3,2} \lambda_{0, k}\right)\right. \\
&\left.+\sum_{h=0}^{i}\left(\Phi_{i-h, M+1}^{4,1} b_{h}+\Phi_{i-h, M+1}^{4,2} \mu_{h, 0}\right)\right)+Z_{i}^{\top} \zeta_{i} .
\end{aligned}
$$

These expressions still lead to a linear relation between $\lambda_{0,0}, \lambda_{0,0}, \lambda_{0,1}, \ldots, \lambda_{0, M}, \mu_{0,0}, \mu_{1,0}, \ldots, \mu_{N, 0}$ and $a_{0}, a_{1}, \ldots, a_{M}, b_{0}, b_{1}, \ldots, b_{N}$, so that equation (26) still holds, but the expression of the matrices $\Omega_{i, j}$ and $\Upsilon_{i, j}$ become more complex.

Remark 5: When $Q=S R^{-1} S^{\top}$, or in the even more particular case of minimum energy control, as considered in [6, Section IV], where $Q$ and $S$ are both zero, the equation (11) and corresponding boundary condition can be solved 'backwards' as described in [6]; this yields the solution presented in [9], which was established via quite different techniques.

\section{ACKNOWLEDGements}

This work was partially supported by the Australian Research Council (Discovery Projects DP0664789 and DP0986577).

\section{REFERENCES}

[1] R. Roesser, "A discrete state-space model for linear image processing," IEEE Transactions on Automatic Control, vol. AC-20, no. 1, pp. 1-10, 1975.

[2] E. Fornasini and G. Marchesini, "Doubly-Indexed Dynamical Systems: State-Space Models and Structural Properties," Mathematical System Theory, vol. 12, pp. 59-72, 1978.

[3] M. Bisiacco and E. Fornasini, "Optimal control of two-dimensional systems," SIAM Journal of Control and Optimization, vol. 28, pp. 582-601, 1990.

[4] M. Bisiacco, "New Results in 2D Optimal Control Theory," Multidimensional Systems and Signal Processing, vol. 6, pp. 189-222, 1995.

[5] A. Ferrante and L. Ntogramatzidis, "Employing the algebraic Riccati equation for a parametrization of the solutions of the finitehorizon LQ problem: the discrete-time case". Systems \& Control Letters, 54:693-703, 2005.

[6] C. Li and M. Fadali, "Optimal control of 2-D systems," IEEE Transactions on Automatic Control, vol. AC-36, no. 2, pp. 223228, February 1991.

[7] O. Mangasarian, "Sufficient conditions for the optimal control of nonlinear systems," SIAM Journal on Control, vol. 4, no. 1, pp. 139-152, 1966.

[8] L. Ntogramatzidis, and M. Cantoni, "On the sufficiency of finite-frame LQR optimality conditions for 2-D Roesser models". In Proceedings of the European Control Conference 2007, Kos, Greece, July 2-5, 2007.

[9] T. Kaczorek and J. Klamka, "Minimum energy control of 2-D linear systems with variable coefficients," International Journal of Control, vol. 44, no. 3, pp. 645-650, 1986. 